\title{
Character Association and Path Analysis of Seed Yield and Its Attributes in Rice as Affected by Bio-Inoculums Under Tropical Environment
}

\author{
${ }^{1}$ R. Bhattacharyya, ${ }^{* 2}$ B. Roy, ${ }^{1}$ M. C. Kabi and ${ }^{1}$ A. K. Basu \\ ${ }^{1}$ Nadule Research Laboratory, Bidhan Chndra Krishi Viswavidyalaya, Mohanpur, Nadia, West \\ Bengal, India \\ ${ }^{2}$ Dept. of Genetics and Plant Breeding, Uttar Banga Krishi Viswavidyalaya, Pundibari, Cooch \\ Behar, West Bengal, India \\ E-mail: bcroy10@yahoo.com
}

Accepted $5^{\text {th }}$ September 2007

\begin{abstract}
Efforts were made to unzip the influence of Azotobacter chroococcum on character association and effects of dependent traits on independent variables on seed yield. Significant positive association of seed yield per plant was noted with number of panicles per plant, number of filled grains per panicle, 1000-seed weight, husked grain length and amylose content in both Azotobacter inoculated and control situations. Fresh weight of shoots per plant at 45 DAT (days after transplanting) showed maximum direct contribution followed by root length towards seed yield per plant in uninoculated condition, but 1000 seed weight exhibited highest contribution followed by number of panicles per plant under inoculated condition. Wide variation was observed for characters association and path coefficient analysis under inoculated and uninoculated conditions.
\end{abstract}

Key words: rice, interrelationship, Azotobacter chroococcum, tropical climate

\section{INTRODUCTION}

Rice is the most important food crop of Asia, Africa and Latin America. Ensuring food security through increased production by adopting intensive agriculture would remain incomplete unless crop production and environmental sustainability would be addressed concurrently. The motivation for the development and safe guard of our environment must be ensured without any conflict, in which management of crop species and cultural practices offer a viable proposition. Indiscriminate use of chemical fertilizers may lead to many biotic and abiotic problems. The requirement and supply of chemical nitrogen fertilizers in rice mismatch in most of the cases resulting loss of 50$70 \%$ of the fertilizer applied. It is thus, imperative to find the ways and means to uplift the present yield level and to optimize the use of various inputs to develop efficient and costeffective system. To overcome this loss of nitrogen application generally two approaches have been suggested: (1) to regulate the timely application of nitrogen on the basis of the re- quirement of the plant and (2) to increase the efficiency of available soil $\mathrm{N}$ and meet the additional requirement by making rice genotype capable of fixing its own nitrogen either directly or through the interaction with Dizatrophic bacteria. As achievement of the later is long term strategy, the adoption and/or restructuring the genotype(s) with desirable characters is much more competent than soil and crop management to the farmers, which may also lead to the necessity of fertilizer application. Hence, it is quite likely to be rational to think of re-orientation of proper nutrient management practices in rice with biofertilizer application so as to save a considerable amount of inorganic inputs being used. Rice is able to utilize a basal level of fixed $\mathrm{N}$ as a source of its nitrogen from $\mathrm{N}$-fixing activities of dizotrophs (Ladha, 1986; Yanni, 1991; Roger and Ladha, 1992). A wide range of diversity of dizotrophs is available, viz., Azotobacter, Azospirillum, Deraxia, Klebsiella, Alkaligens etc. which are well known. Diazotrophs fix atmospheric $\mathrm{N}$ in soil by reducing it to ammonium through electron reduction and protonation of gaseous dini-

\footnotetext{
*Corresponding author
} 
trogen. Nitrogenase enzyme complex interacts with this fixation procedure the biosynthesis of nitrogenase enzyme is determined by $15-20$ different nitrogen fixing (nif) gene products (Dean and Jacobson, 1992). Keeping in view the potentials of $\mathrm{N}$-fixers as biofertilizer in the integrated nutrient management system and plant genomes are likely to contribute to a greater extent in recycling atmospheric nitrogen as well as the utilization of merits of genotype specific interaction with the dizotrophs, it seems relevant to undertake the present investigation in order to find out the pattern of screening rice genotypes with $\mathrm{N}$-fixing efficiencies through effective breeding techniques in response to inoculation with Azotobacter for association among different attributes of seed yield.

\section{MATERIALS AND METHODS}

The experiment was conducted at District Seed Farm, Kalyani and Chakda, Regional Research Station, Bidhan Chandra Krishi Viswavidyalaya during Kharif (wet) season of two consecutive years. The experimental materials were comprised of 12 genotypes including 2 local cultivars, 10 high yielding varieties. Seedlings at 21 days after germination were treated by dipping their roots in carrier-based slurry of A. chroococcum for 30 minutes before transplanting. The width of individual block was $3 \mathrm{~m}$ and there were three replications. Spacing was maintained as $20 \mathrm{~cm}{ }^{\prime} 15 \mathrm{~cm}$. The experimental design followed was split-plot, treatments (biofertilizer) were placed in subplots and varieties were assigned as main plots. Single seedling per hill was transplanted. Standard agronomic practices compatible to this

Table 1a: Genotypic (G) and phenotypic (P) correlation coefficient amongst eight characters control set of experiment

\begin{tabular}{|c|c|c|c|c|c|c|c|c|c|c|c|c|c|c|c|c|}
\hline$\overline{\text { Trait } X_{2}}$ & $\overline{\mathbf{X}_{3}}$ & $\mathbf{X}_{4}$ & $\overline{X_{5}}$ & $\overline{X_{6}}$ & $\mathrm{X}_{7}$ & $\mathbf{X}_{8}$ & $\mathbf{X}_{9}$ & $\overline{X_{10}}$ & $\overline{X_{11}}$ & $\overline{X_{12}}$ & $\mathbf{X}_{13}$ & $\overline{X_{14}}$ & $\overline{X_{15}}$ & $\mathbf{X}_{16}$ & $\overline{X_{17}}$ & $\overline{X_{18}}$ \\
\hline$\overline{X_{1} G 0.563}$ & 0.092 & -0.449 & -0.587 & -0.343 & 0.104 & 0.423 & -0.137 & -0.350 & -0.401 & 0.592 & 0.603 & -0.400 & 0.362 & 0.410 & 0.076 & $\overline{-0.414}$ \\
\hline $\mathrm{P} 0.511^{* *}$ & 0.057 & $-0.343 *$ & $-0.417^{*}$ & -0.265 & 0.141 & 0.311 & 0.131 & -0.233 & -0.242 & $0.489 * *$ & $* 0.665 * *$ & 0.123 & 0.420 & $0.383^{*}$ & 0.133 & -0.084 \\
\hline $\mathrm{X}_{2} \mathrm{G}$ & -0.255 & -0.469 & -0.240 & -0.214 & 0.002 & 0.101 & 0.696 & -0.306 & -0.069 & 0.955 & 0.754 & -0.633 & 0.615 & 0.230 & 0.403 & 0.055 \\
\hline $\mathrm{P}$ & -0.236 & $-0.452 * *$ & -0.238 & -0.211 & 0.016 & 0.094 & $0.397^{*}$ & -0.263 & -0.056 & $0.935 * *$ & $* 0.699 * *$ & 0.036 & $0.598 * *$ & 0.245 & $0.349^{*}$ & 0.059 \\
\hline $\mathrm{X}_{3} \mathrm{G}$ & & 0.421 & 0.263 & 0.400 & 0.016 & 0.560 & 0.108 & -0.581 & 0.636 & -0.213 & 0.157 & -0.063 & 0.321 & 0.518 & 0.403 & -0.457 \\
\hline $\mathrm{P}$ & & $0.413 *$ & 0.252 & $0.386^{*}$ & 0.235 & $0.543 * *$ & ${ }^{*} 0.015$ & $-0.453^{* *}$ & $0.605^{* *}$ & -0.204 & 0.138 & -0.064 & 0.274 & $0.478^{* *}$ & 0.320 & -0.293 \\
\hline $\mathrm{X}_{4} \mathrm{G}$ & & & 0.489 & 0.395 & 0.203 & 0.276 & 0.012 & 0.004 & 0.457 & -0.426 & -0.324 & 0.832 & -0.106 & -0.193 & 0.099 & 0.427 \\
\hline $\mathrm{P}$ & & & $0.479 * *$ & $0.931 * *$ & $* 0.321$ & 0.266 & 0.020 & 0.009 & $0.433^{* *}$ & -0.419 & -0.282 & 0.143 & -0.091 & -0.176 & 0.085 & 0.294 \\
\hline $\mathrm{X}_{5} \mathrm{G}$ & & & & 0.425 & 0.271 & 0.084 & 0.165 & 0.213 & 0.348 & -0.211 & -0.370 & 0.887 & -0.279 & -0.387 & -0.109 & 0.039 \\
\hline $\mathrm{P}$ & & & & $0.418 * *$ & * 0.284 & 0.086 & 0.083 & 0.172 & 0.319 & -0.211 & -0.290 & 0.126 & -0.249 & $-0.357^{*}$ & -0.085 & 0.035 \\
\hline $\mathrm{X}_{6} \mathrm{G}$ & & & & & 0.496 & 0.392 & 0.391 & -0.001 & 0.428 & -0.138 & -0.179 & 0.297 & 0.107 & -0.064 & 0.269 & 0.532 \\
\hline $\mathrm{P}$ & & & & & 0.407 * & $* 0.392 *$ & 0.186 & -0.011 & $0.405^{*}$ & -0.139 & -0.154 & 0.217 & 0.082 & -0.064 & 0.211 & $0.372 *$ \\
\hline $\mathrm{X}_{7} \mathrm{G}$ & & & & & & 0.770 & 0.380 & 0.444 & -0.107 & 0.178 & -0.348 & 0.439 & 0.192 & 0.185 & 0.036 & 0.227 \\
\hline $\mathrm{P}$ & & & & & & 0.621 & 0.528 ** & $0.501 * *$ & -0.064 & 0.172 & -0.204 & $0.425^{* *}$ & 0.248 & 0.234 & 0.131 & 0.173 \\
\hline $\mathrm{X}_{8} \mathrm{G}$ & & & & & & & 0.083 & -0.215 & 0.028 & 0.160 & 0.233 & 0.922 & 0.648 & 0.684 & 0.608 & 0.327 \\
\hline $\mathrm{P}$ & & & & & & & 0.044 & -0.184 & 0.019 & 0.158 & 0.197 & 0.120 & $0.555^{* *}$ & $0.639 * *$ & $0.495 * *$ & 0.225 \\
\hline $\mathrm{X}_{9} \mathrm{G}$ & & & & & & & & 0.058 & 0.367 & 0.806 & 0.086 & 0.598 & 0.383 & -0.025 & -0.020 & -0.156 \\
\hline $\mathrm{P}$ & & & & & & & & 0.115 & 0.207 & $0.449 * *$ & ${ }^{*} 0.170$ & 0.251 & $0.378^{*}$ & 0.139 & 0.117 & -0.030 \\
\hline $\mathrm{X}_{10} \mathrm{G}$ & & & & & & & & & -0.517 & -0.154 & -0.831 & 0.742 & -0.631 & -0.576 & -0.696 & 0.268 \\
\hline $\mathrm{P}$ & & & & & & & & & $-0.395^{*}$ & -0.120 & $-0.623^{* *}$ & $0.419 *$ & $-0.463^{* *}$ & -0.426 & -0.388 & 0.139 \\
\hline $\mathrm{X}_{11} \mathrm{G}$ & & & & & & & & & & -0.121 & -0.004 & -0.468 & 0.205 & 0.200 & 0.474 & -0.306 \\
\hline $\mathrm{P}$ & & & & & & & & & & 0.104 & 0.031 & -0.164 & 0.182 & 0.179 & 0.340 & -0.251 \\
\hline $\mathrm{X}_{12} \mathrm{G}$ & & & & & & & & & & & 0.676 & 0.319 & 0.547 & 0.270 & 0.341 & 0.056 \\
\hline $\mathrm{P}$ & & & & & & & & & & & $0.591 * *$ & 0.114 & $0.493 * *$ & 0.270 & 0.275 & 0.031 \\
\hline $\mathrm{X}_{13} \mathrm{G}$ & & & & & & & & & & & & -0.702 & 0.704 & 0.433 & 0.523 & -0.152 \\
\hline $\mathrm{P}$ & & & & & & & & & & & & -0.101 & 0.643 & $0.396^{*}$ & $0.421 *$ & 0.059 \\
\hline $\mathrm{X}_{14} \mathrm{G}$ & & & & & & & & & & & & & 0.095 & -0.012 & 0.669 & 0.385 \\
\hline $\mathrm{P}$ & & & & & & & & & & & & & 0.025 & 0.039 & 0.049 & 0.151 \\
\hline $\mathrm{X}_{15} \mathrm{G}$ & & & & & & & & & & & & & & 0.734 & 0.783 & 0.264 \\
\hline $\mathrm{P}$ & & & & & & & & & & & & & & $0.737 *$ & $0.714 *$ & 0.170 \\
\hline $\mathrm{X}_{16} \mathrm{G}$ & & & & & & & & & & & & & & & 0.683 & -0.022 \\
\hline P & & & & & & & & & & & & & & & $0.653 * *$ & -0.043 \\
\hline $\mathrm{X}_{17} \mathrm{G}$ & & & & & & & & & & & & & & & & 0.433 \\
\hline$P$ & & & & & & & & & & & & & & & & 0.024 \\
\hline
\end{tabular}

$\mathrm{X}_{1}$ : Plant height, $\mathrm{X}_{2}$ : Panicle length, $\mathrm{X}_{3}$ : No. of panicle/plant, $\mathrm{X}_{4}$ : No. of filled grains/panicle, $\mathrm{X}_{5}$ : 1000 -seeds weight, $\mathrm{X}_{6}$ : Seed yield per plant, $\mathrm{X}_{7}$ : Husked grain length, $\mathrm{X}_{8}$ : Husked grain width, $\mathrm{X}_{9}$ : Dehusked grain length, $\mathrm{X}_{10}$ : Dehusked grain width, $\mathrm{X}_{11}$ : Shoot length at 45 DAT, $X_{12}$ : Shoot fresh wt. at 45 DAT, $X_{13}$ : Shoot dry wt. at 45 DAT, $_{14}$ : Tiller per plant at 45 DAT, $X_{15}$ : Root length at 45 DAT, $\mathrm{X}_{16}$ : Root fresh wt. at 45 DAT, $\mathrm{X}_{17}$ : Root dry wt. at 45 DAT, $\mathrm{X}_{18}$ : Amylose content, G: Genotypic coefficient of variance, P: Phenotypic coefficient of variance, G: Genotypic coefficient of variance, P: Phenotypic coefficient of variance, *, **: Significant at $5 \%$ and $1 \%$ levels, respectively 
Table 1b: Genotypic (G) and phenotypic (P) correlation coefficient amongst eight characters Azotobacter inoculated set of experiment

\begin{tabular}{|c|c|c|c|c|c|c|c|c|c|c|c|c|c|c|c|c|c|}
\hline Trait & $\mathrm{X}_{2}$ & $\mathrm{X}_{3}$ & $\mathrm{X}_{4}$ & $\mathrm{X}_{5}$ & $\mathrm{X}_{6}$ & $\mathrm{X}_{7}$ & $\mathrm{X}_{8}$ & $\mathrm{X}_{9}$ & $\mathrm{X}_{10}$ & $\mathrm{X}_{11}$ & $\mathrm{X}_{12}$ & $X_{13}$ & $\mathrm{X}_{14}$ & $\mathrm{X}_{15}$ & $\mathrm{X}_{16}$ & $\mathrm{X}_{17}$ & $\mathrm{X}_{18}$ \\
\hline$\overline{\mathrm{X}_{1}}$ & $\begin{array}{ll}G & -0.159 \\
P & -0.132\end{array}$ & $\begin{array}{l}-0.234 \\
-0.193\end{array}$ & $\begin{array}{l}0.619 \\
0.414^{*}\end{array}$ & $\begin{array}{l}0.163 \\
0.159\end{array}$ & $\begin{array}{l}0.232 \\
0.189\end{array}$ & $\begin{array}{l}-0.133 \\
-0.130\end{array}$ & $\begin{array}{l}-0.213 \\
-0.211\end{array}$ & $\begin{array}{l}-0.425 \\
-0.421 \\
*\end{array}$ & $\begin{array}{l}-0.206 \\
-0.205\end{array}$ & $\begin{array}{l}0.970 \\
0.938 \\
* *\end{array}$ & $\begin{array}{l}0.668 \\
0.492 \\
* *\end{array}$ & $\begin{array}{l}0.653 \\
0.598 \\
* *\end{array}$ & $\begin{array}{l}0.196 \\
0.071\end{array}$ & $\begin{array}{l}0.452 \\
0.370 \\
*\end{array}$ & $\begin{array}{l}0.310 \\
0.293\end{array}$ & $\begin{array}{l}0.351 \\
0.287\end{array}$ & $\begin{array}{l}0.657 \\
0.518 \\
* *\end{array}$ \\
\hline $\mathrm{X}_{2}$ & $\begin{array}{l}\mathrm{G} \\
\mathrm{P}\end{array}$ & $\begin{array}{l}-0.611 \\
-0.422 \\
* *\end{array}$ & $\begin{array}{l}0.199 \\
0.211\end{array}$ & $\begin{array}{l}0.036 \\
0.033\end{array}$ & $\begin{array}{l}-0.097 \\
-0.051\end{array}$ & $\begin{array}{l}0.452 \\
0.434 \\
* *\end{array}$ & $\begin{array}{l}0.298 \\
0.298\end{array}$ & $\begin{array}{l}0.485 \\
0.469 \\
* *\end{array}$ & $\begin{array}{l}0.641 \\
0.604 \\
* *\end{array}$ & $\begin{array}{l}-0.101 \\
-0.003\end{array}$ & $\begin{array}{l}-0.339 \\
-0.221\end{array}$ & $\begin{array}{l}0.143 \\
0.119\end{array}$ & $\begin{array}{l}-0.843 \\
-0.306\end{array}$ & $\begin{array}{l}0.288 \\
0.227\end{array}$ & $\begin{array}{l}0.256 \\
0.222\end{array}$ & $\begin{array}{l}0.444 \\
0.376 \\
*\end{array}$ & $\begin{array}{l}-0.245 \\
-0.164\end{array}$ \\
\hline$X_{3}$ & $\begin{array}{l}\mathrm{G} \\
\mathrm{P}\end{array}$ & & $\begin{array}{l}0.046 \\
-0.004\end{array}$ & $\begin{array}{l}-0.204 \\
-0.152\end{array}$ & $\begin{array}{l}0.277 \\
0.390^{*}\end{array}$ & $\begin{array}{l}0.044 \\
0.029\end{array}$ & $\begin{array}{l}0.166 \\
0.105\end{array}$ & $\begin{array}{l}0.070 \\
0.038\end{array}$ & $\begin{array}{l}-0.627 \\
-0.415^{*}\end{array}$ & $\begin{array}{l}-0.356 \\
-0.305\end{array}$ & $\begin{array}{l}-0.416 \\
-0.303\end{array}$ & $\begin{array}{l}-0.888 \\
-0.648 \\
* *\end{array}$ & $\begin{array}{l}0.468 \\
-0.390 \\
*\end{array}$ & $\begin{array}{l}-0.515 \\
-0.422\end{array}$ & $\begin{array}{l}-0.543 \\
-0.439 \\
* *\end{array}$ & $\begin{array}{l}-0.643 \\
-0.432 \\
* *\end{array}$ & $\begin{array}{l}-0.014 \\
-0.062\end{array}$ \\
\hline $\mathrm{X}_{4}$ & $\begin{array}{l}\mathrm{G} \\
\mathrm{P}\end{array}$ & & & $\begin{array}{l}0.110 \\
0.077\end{array}$ & $\begin{array}{l}0.542 \\
0.531 \\
* *\end{array}$ & $\begin{array}{l}0.431 \\
0.273\end{array}$ & $\begin{array}{l}0.056 \\
0.062\end{array}$ & $\begin{array}{l}0.123 \\
0.062\end{array}$ & $\begin{array}{l}0.110 \\
0.092\end{array}$ & $\begin{array}{l}0.525 \\
0.319\end{array}$ & $\begin{array}{l}0.274 \\
-0.042\end{array}$ & $\begin{array}{l}0.198 \\
0.034\end{array}$ & $\begin{array}{l}0.259 \\
0.139\end{array}$ & $\begin{array}{l}0.300 \\
0.087\end{array}$ & $\begin{array}{l}0.078 \\
-0.021\end{array}$ & $\begin{array}{l}-0.050 \\
-0.014\end{array}$ & $\begin{array}{l}0.742 \\
0.367 \\
*\end{array}$ \\
\hline$X_{5}$ & $\begin{array}{l}\mathrm{G} \\
\mathrm{P}\end{array}$ & & & & $\begin{array}{l}0.783 \\
0.698 \\
* *\end{array}$ & $\begin{array}{l}0.384 \\
0.382 \\
*\end{array}$ & $\begin{array}{l}0.082 \\
0.081\end{array}$ & $\begin{array}{l}0.268 \\
0.267\end{array}$ & $\begin{array}{l}0.574 \\
0.553 \\
* *\end{array}$ & $\begin{array}{l}0.129 \\
0.123\end{array}$ & $\begin{array}{l}0.544 \\
0.366 \\
*\end{array}$ & $\begin{array}{l}0.257 \\
0.221\end{array}$ & $\begin{array}{l}0.193 \\
0.074\end{array}$ & $\begin{array}{l}0.651 \\
0.504 \\
* *\end{array}$ & $\begin{array}{l}0.678 \\
0.626 \\
* *\end{array}$ & $\begin{array}{l}0.556 \\
0.541 \\
* *\end{array}$ & $\begin{array}{l}0.340 \\
0.273\end{array}$ \\
\hline $\mathrm{X}_{6}$ & $\begin{array}{l}\mathrm{G} \\
\mathrm{P}\end{array}$ & & & & & $\begin{array}{l}0.524 \\
0.454 \\
* *\end{array}$ & $\begin{array}{l}0.154 \\
0.135\end{array}$ & $\begin{array}{l}0.343 \\
0.285\end{array}$ & $\begin{array}{l}0.308 \\
0.304\end{array}$ & $\begin{array}{l}0.107 \\
0.066\end{array}$ & $\begin{array}{l}0.394 \\
0.119\end{array}$ & $\begin{array}{l}-0.137 \\
-0.170\end{array}$ & $\begin{array}{l}0.886 \\
0.333 \\
*\end{array}$ & $\begin{array}{l}0.392 \\
0.182\end{array}$ & $\begin{array}{l}0.340 \\
0.214\end{array}$ & $\begin{array}{l}0.133 \\
0.069\end{array}$ & $\begin{array}{l}0.568 \\
0.382 \\
*\end{array}$ \\
\hline$X_{7}$ & $\begin{array}{l}\mathrm{G} \\
\mathrm{P}\end{array}$ & & & & & & $\begin{array}{l}0.434 \\
0.429 \\
* *\end{array}$ & $\begin{array}{l}0.933 \\
0.929 \\
* *\end{array}$ & $\begin{array}{l}0.384 \\
0.364^{*}\end{array}$ & $\begin{array}{l}-0.211 \\
-0.203\end{array}$ & $\begin{array}{l}-0.258 \\
-0.173\end{array}$ & $\begin{array}{l}-0.110 \\
-0.089\end{array}$ & $\begin{array}{l}-0.404 \\
0.162\end{array}$ & $\begin{array}{l}0.170 \\
0.139\end{array}$ & $\begin{array}{l}-0.026 \\
-0.019\end{array}$ & $\begin{array}{l}-0.238 \\
0.201\end{array}$ & $\begin{array}{l}0.238 \\
0.250\end{array}$ \\
\hline $\mathrm{X}_{8}$ & $\begin{array}{l}\mathrm{G} \\
\mathrm{P}\end{array}$ & & & & & & & $\begin{array}{l}0.489 \\
0.484 \\
* *\end{array}$ & $\begin{array}{l}0.275 \\
0.253\end{array}$ & $\begin{array}{l}-0.252 \\
-0.251\end{array}$ & $\begin{array}{l}-0.434 \\
-0.335 \\
*\end{array}$ & $\begin{array}{l}-0.289 \\
-0.268\end{array}$ & $\begin{array}{l}0.115 \\
0.076\end{array}$ & $\begin{array}{l}-0.316 \\
-0.274\end{array}$ & $\begin{array}{l}-0.408 \\
-0.383 \\
*\end{array}$ & $\begin{array}{l}-0.158 \\
-0.142\end{array}$ & $\begin{array}{l}0.044 \\
0.019\end{array}$ \\
\hline $\mathrm{X}_{9}$ & $\begin{array}{l}\mathrm{G} \\
\mathrm{P}\end{array}$ & & & & & & & & $\begin{array}{l}0.417 \\
0.395^{*}\end{array}$ & $\begin{array}{l}-0.470 \\
-0.456 \\
* *\end{array}$ & $\begin{array}{l}-0.364 \\
-0.254\end{array}$ & $\begin{array}{l}-0.245 \\
-0.222\end{array}$ & $\begin{array}{l}0.170 \\
0.095\end{array}$ & $\begin{array}{l}0.008 \\
0.015\end{array}$ & $\begin{array}{l}-0.155 \\
-0.135\end{array}$ & $\begin{array}{l}0.093 \\
0.092\end{array}$ & $\begin{array}{l}0.145 \\
0.123\end{array}$ \\
\hline $\mathrm{X}_{10}$ & $\begin{array}{l}\mathrm{G} \\
\mathrm{P}\end{array}$ & & & & & & & & & $\begin{array}{l}-0.236 \\
-0.223\end{array}$ & $\begin{array}{l}0.238 \\
0.148\end{array}$ & $\begin{array}{l}-0.231 \\
0.188\end{array}$ & $\begin{array}{l}-0.573 \\
-0.166\end{array}$ & $\begin{array}{l}0.308 \\
0.204\end{array}$ & $\begin{array}{l}-0.463 \\
0.389 \\
*\end{array}$ & $\begin{array}{l}0.368 \\
0.269\end{array}$ & $\begin{array}{l}0.010 \\
0.025\end{array}$ \\
\hline$X_{11}$ & $\begin{array}{l}\mathrm{G} \\
\mathrm{P}\end{array}$ & & & & & & & & & & $\begin{array}{l}0.618 \\
0.540\end{array}$ & $\begin{array}{l}0.715 \\
0.688 \\
* *\end{array}$ & $\begin{array}{l}-0.171 \\
-0.031\end{array}$ & $\begin{array}{l}0.525 \\
0.509 \\
* *\end{array}$ & $\begin{array}{l}0.310 \\
0.330\end{array}$ & $\begin{array}{l}0.453 \\
0.383 \\
*\end{array}$ & $\begin{array}{l}0.585 \\
0.451 \\
* *\end{array}$ \\
\hline$X_{12}$ & $\begin{array}{l}\mathrm{G} \\
\mathrm{P}\end{array}$ & & & & & & & & & & & $\begin{array}{l}0.653 \\
0.684 \\
* *\end{array}$ & $\begin{array}{l}-0.222 \\
0.017\end{array}$ & $\begin{array}{l}0.389 \\
0.392 \\
*\end{array}$ & $\begin{array}{l}0.426 \\
0.365 \\
*\end{array}$ & $\begin{array}{l}0.229 \\
0.179\end{array}$ & $\begin{array}{l}0.833 \\
0.509 \\
* *\end{array}$ \\
\hline$X_{13}$ & $\begin{array}{l}\mathrm{G} \\
\mathrm{P}\end{array}$ & & & & & & & & & & & & $\begin{array}{l}-0.868 \\
-0.271\end{array}$ & $\begin{array}{l}0.694 \\
0.591 \\
* *\end{array}$ & $\begin{array}{l}0.506 \\
0.472 \\
* *\end{array}$ & $\begin{array}{l}0.676 \\
0.517 \\
* *\end{array}$ & $\begin{array}{l}0.488 \\
0.320\end{array}$ \\
\hline $\mathrm{X}_{14}$ & $\begin{array}{l}\mathrm{G} \\
\mathrm{P}\end{array}$ & & & & & & & & & & & & & $\begin{array}{l}-0.728 \\
-0.169\end{array}$ & $\begin{array}{l}-0.522 \\
-0.128\end{array}$ & $\begin{array}{l}-0.643 \\
-0.101\end{array}$ & $\begin{array}{l}0.530 \\
-0.026\end{array}$ \\
\hline$X_{15}$ & $\begin{array}{l}\mathrm{G} \\
\mathrm{P}\end{array}$ & & & & & & & & & & & & & & $\begin{array}{l}0.800 \\
0.794 \\
* *\end{array}$ & $\begin{array}{l}0.897 \\
0.782 \\
* *\end{array}$ & $\begin{array}{l}0.284 \\
0.147\end{array}$ \\
\hline$X_{16}$ & $\begin{array}{l}\mathrm{G} \\
\mathrm{P}\end{array}$ & & & & & & & & & & & & & & & $\begin{array}{l}0.725 \\
0.691 \\
* *\end{array}$ & $\begin{array}{l}-0.013 \\
-0.064\end{array}$ \\
\hline$X_{17}$ & $\begin{array}{l}\mathrm{G} \\
\mathrm{P}\end{array}$ & & & & & & & & & & & & & & & & $\begin{array}{l}-0.048 \\
-0.076 \\
\end{array}$ \\
\hline
\end{tabular}

$\mathrm{X}_{1}$ : Plant height, $\mathrm{X}_{2}$ : Panicle length, $\mathrm{X}_{3}$ : No. of panicle/plant, $\mathrm{X}_{4}$ : No. of filled grains/panicle, $\mathrm{X}_{5}$ : 1000-seeds weight, $\mathrm{X}_{6}$ : Seed yield per plant, $X_{7}$ : Husked grain length, $X_{8}$ : Husked grain width, $X_{9}$ : Dehusked grain length, $X_{10}$ : Dehusked grain width, $X_{11}$ : Shoot length at 45 DAT, $X_{12}$ : Shoot fresh wt. at 45 DAT, $X_{13}$ : Shoot dry wt. at 45 DAT, $X_{14}$ : Tiller per plant at 45 DAT, $X_{15}$ : Root length at 45 DAT, $X_{16}$ : Root fresh wt. at 45 DAT, $X_{17}$ : Root dry wt. at 45 DAT, $X_{18}$ : Amylose content, G: Genotypic coefficient of variance, P: Phenotypic coefficient of variance, G: Genotypic coefficient of variance, P: Phenotypic coefficient of variance, *, **: Significant at $5 \%$ and $1 \%$ levels, respectively

ago-ecological zone were adopted to ensure good crop growth. Observations were recorded on plant height, panicle length, number of panicle per plant, number of filled grains per panicle, 1000-seeds weight, seed yield per plant, husked grain length, husked grain width, dehusked grain length, dehusked grain width, shoot length at 45 DAT, shoot fresh weight at 45 DAT, shoot dry weight at 45 DAT, tiller per plant at $45 \mathrm{DAT}$, root length at $45 \mathrm{DAT}$, root fresh weight at $45 \mathrm{DAT}$, root dry weight at 45 DAT, and amylose content. The amylose con- tent was determined for each genotype from the freshly harvested seeds using the method described by Sadasivam and Monica (1996). The mean performance of individual genotype over two years was pooled and employed for statistical analysis. Both phenotypic and genotypic correlation coefficients were worked out following the method outlined by Al-Jibuori et al. (1958). The direct and indirect effects of individual characters on seed yield were estimated using the method suggested by Dewey and $\mathrm{Lu}$ (1959). 


\section{RESULTS AND DISCUSSION}

Selection based on the detailed knowledge of magnitude and direction of association between yield and its attributes is very important in identifying the key characters, which can be exploited for crop improvement through suitable breeding programme.

Both the genotypic and phenotypic correlation coefficients among 18 characters have been presented in Tables $1 \mathrm{a}$ and $1 \mathrm{~b}$ for uninoculated and inoculated conditions, respectively. Under both situations, the genotypic correlation coefficients were higher than the phenotypic correlation coefficient, indicating the predominant role of genetic background rather than environmental effect for association between different characters. These findings are corroborating the observations of Meenakshi et al. (1999), Chaubey and Singh (1994).
Seed yield per plant showed positive and significant association with number of panicles per plant, number of filled grains per panicle, 1000-seed weight, husked grain length and amylose content in both the situation. Chakraborty et al. (2001) and Yogamenakshi et al. (2004) also reported significant positive correlation of grain yield per plant with plant height, number of panicle per plant, panicle length and number of filled grains per panicle. In addition to those, positive significant association of seed yield per plant was noted with husked grain width and shoot length at 45 DAT in control and with number of tillers per plant at 45 DAT under inoculated condition. Number of panicles per plant was found to be significantly correlated in positive direction with number of filled grains per panicle, husked grain width, shoot length at 45 DAT, and root fresh weight at 45 DAT in control

Table 2 Direct and indirect effects of different characters on seed yield per plant

\begin{tabular}{|c|c|c|c|c|c|c|c|c|c|c|c|c|c|c|c|c|c|}
\hline Trait & & $\overline{X_{1}}$ & $\overline{X_{2}}$ & $X_{3}$ & $\mathrm{X}_{4}$ & $\mathrm{X}_{5}$ & $\mathrm{X}_{6}$ & $\mathbf{X}_{7}$ & $\mathbf{X}_{8}$ & $X_{9}$ & $\mathbf{X}_{10}$ & $X_{11}$ & $\mathrm{X}_{12}$ & $\overline{X_{13}}$ & $\mathrm{X}_{14}$ & $\mathrm{X}_{15}$ & $X_{16}$ \\
\hline \multirow[t]{2}{*}{$\overline{X_{1}}$} & $\mathrm{C}_{0}$ & 0.346 & -1.895 & -0.038 & -0.301 & -0.078 & 0.019 & -0.014 & 0.091 & -0.271 & -0.522 & 1.544 & 0.425 & 0.651 & -0.368 & -0.007 & 0.120 \\
\hline & $\mathrm{C}_{1}$ & 0.058 & -0.011 & -0.110 & 0.188 & 0.118 & 0.016 & 0.02 & -0.071 & -0.048 & 0.144 & 0.041 & -0.134 & 0.044 & -0.027 & -0.017 & .015 \\
\hline \multirow[t]{2}{*}{$\mathrm{X}_{2}$} & $\mathrm{C}_{0}$ & 0.195 & -3.366 & 0.229 & & & 0.000 & -0.003 & -0.046 & & -0.089 & 2.489 & 531 & 1.105 & -0.206 & -0.040 & 0.016 \\
\hline & $\mathrm{C}_{1}$ & -0.009 & 0.071 & -0.289 & 0.060 & 0.026 & -0.055 & -0.034 & 0.081 & 0.151 & -0.015 & -0.021 & -0.029 & 0.028 & -0.023 & -0.021 & -0.006 \\
\hline \multirow[t]{2}{*}{$X_{3}$} & $\mathrm{C}_{0}$ & 0.032 & 0.857 & -0.900 & 281 & 0.035 & 0.044 & -0.018 & -0.071 & -0.449 & 0.830 & -0.554 & 0.110 & 0.576 & -0.464 & -0.040 & .133 \\
\hline & $\begin{array}{l}\mathrm{C}_{1} \\
\mathrm{C}_{0}\end{array}$ & -0.155 & $\begin{array}{l}-0.044 \\
1.578\end{array}$ & $\begin{array}{l}\mathbf{0 . 4 7 3} \\
-0.377\end{array}$ & 0.671 & $\begin{array}{l}-0.147 \\
0.065\end{array}$ & $\begin{array}{l}-0.005 \\
0.060\end{array}$ & $\begin{array}{l}-0.019 \\
-0.009\end{array}$ & 0,008 & $\begin{array}{l}-0.148 \\
0.003\end{array}$ & $\begin{array}{l}-0.053 \\
0.597\end{array}$ & $\begin{array}{l}-0.026 \\
-1.111\end{array}$ & $\begin{array}{l}0.182 \\
-0228\end{array}$ & $\begin{array}{l}-0.051 \\
-0.191\end{array}$ & 0.173 & $-0,010$ & $\begin{array}{l}0.000 \\
-0.124\end{array}$ \\
\hline$X_{4}$ & $\mathrm{C}_{1}$ & 0.036 & 14 & . & 0.303 & 79 & 3 & -0.006 & 0.021 & 0.026 & 0.078 & 0.017 & -0.041 & 0.029 & -0.007 & 0.002 & 0.017 \\
\hline \multirow[t]{2}{*}{$\mathrm{X}_{5}$} & $\mathrm{C}_{0}$ & -0.203 & 0.808 & -0.236 & 328 & 132 & 053 & -0.003 & 109 & & 454 & -0.550 & -0.260 & -0.501 & 0.347 & 0.011 & -0.011 \\
\hline & $\mathrm{C}_{1}$ & 0.009 & 0.003 & -0.096 & 0.033 & 22 & -0.047 & -0.009 & 0.0 & 5 & 0.019 & 0.034 & -0.053 & 0.064 & -0.060 & -0.027 & 0.008 \\
\hline \multirow[t]{2}{*}{$X_{6}$} & $\mathrm{C}_{0}$ & 0.036 & -0.008 & -0.212 & 216 & 038 & 0.187 & -0.024 & -0.251 & 343 & -0.139 & 0.4 & -0.245 & 0.345 & -0.166 & -0.004 & 0.086 \\
\hline & $\mathrm{C}_{1}$ & -0.008 & 0.032 & & 131 & 271 & & -0.049 & & 0.0 & -0.031 & -0.016 & .023 & 0.017 & 0.002 & -0.011 & .007 \\
\hline \multirow[t]{2}{*}{$X_{7}$} & $\mathrm{C}_{0}$ & 0.146 & -0.342 & -0.504 & 0.179 & 0.011 & 0.137 & -0.033 & -0.055 & -0.167 & 0.036 & 0.421 & 0.164 & 1.166 & -0.613 & -0.060 & -0.095 \\
\hline & $\mathrm{C}_{1}$ & -0.012 & 0.021 & 0.078 & 017 & .059 & -0.053 & -0.113 & 0.081 & 0.065 & -0.037 & -0.027 & 0.059 & -0.031 & 0.036 & 0.008 & 0.001 \\
\hline \multirow[t]{2}{*}{$\mathrm{X}_{8}$} & $\mathrm{C}_{0}$ & -0.048 & -2.344 & -0.097 & 0.008 & 0.022 & 0.071 & -0.003 & -0.661 & 5 & 0.478 & 2.101 & 0.066 & 0.688 & 0.022 & 0.002 & 0.045 \\
\hline & $\mathrm{C}_{1}$ & 025 & 0.035 & 0.033 & 037 & 193 & 14 & -0.055 & & ( & -0.070 & 022 & 0 & 0.001 & 0.014 & 04 & 0.003 \\
\hline \multirow[t]{2}{*}{$\mathrm{X}_{9}$} & $\mathrm{C}_{0}$ & -0 & 30 & 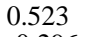 & 0.003 & 0.028 & & 0.007 & & 0.7 & 54 & 2 & 35 & -1.135 & 0.516 & 0.068 & -0.078 \\
\hline & $\mathrm{C}_{1}$ & -0.012 & 046 & -0.296 & 0.033 & 0.415 & .047 & -0.031 & & 0.236 & -0.035 & 0.015 & -0.047 & 0.030 & -0.041 & -0.018 & 0.000 \\
\hline \multirow[t]{2}{*}{$\mathrm{X}_{10}$} & $\mathrm{C}_{0}$ & -0.139 & 0.231 & -0.572 & $\begin{array}{l}307 \\
150\end{array}$ & 0.046 & -0.020 & -0.001 & -0 & $\begin{array}{l}-0.399 \\
-0.56\end{array}$ & $\begin{array}{l}1.304 \\
0.148\end{array}$ & -0.316 & -0.003 & 0.368 & -0.179 & -0.047 & 0.089 \\
\hline & $\mathrm{C}_{1}$ & 56 & -0.007 & 68 & 59 & .093 & 26 & 0.028 & 78 & -0 & 0.148 & 0.0 & 47 & 0.052 & -0.027 & -0.022 & 0.013 \\
\hline \multirow[t]{2}{*}{$X_{11}$} & $\mathrm{C}_{0}$ & 0.205 & -3.213 & 0.191 & .286 & .028 & 0.033 & -0.005 & -0.533 & -0.119 & -0.158 & 2.607 & 0.476 & 0.983 & -0.242 & -0.033 & -0.016 \\
\hline & $\mathrm{C}_{1}$ & & & & & & & 0. & & & 22 & 62 & 34 & 38 & 38 & & 019 \\
\hline \multirow{2}{*}{$X_{12}$} & $\mathrm{C}_{0}$ & 99 & -2.540 & -0 & 7 & 9 & 65 & 8 & -0.576 & & 05 & 1.762 & 0 & 66 & 88 & 551 & 0.044 \\
\hline & $\mathrm{C}_{1}$ & 0.038 & 0.010 & -0.420 & 0.060 & 0.185 & 0.013 & 0.033 & -0.041 & 0.054 & 0.106 & 0.040 & -0.206 & 0.068 & -0.045 & -0.032 & 0.011 \\
\hline \multirow[t]{2}{*}{$X_{13}$} & $\mathrm{C}_{0}$ & 0.125 & -2.072 & -0.2 & -0.071 & -0.037 & 0.03 & -0.021 & -0.253 & -0.488 & 0.267 & 1.426 & 0.496 & 1.7 & -0.658 & -0.077 & -0.077 \\
\hline & & & 21 & & & 470 & 0.021 & 0036 & 0.00 & & 0.078 & $00 ?$ & & 0098 & 0 & 43 & 0006 \\
\hline \multirow[t]{2}{*}{$\mathrm{X}_{14}$} & $\mathrm{C}_{0}$ & 0.142 & -0.774 & -0.466 & .129 & 0.051 & 0.035 & -0.022 & 0.0 & -0.445 & 0.260 & 0.704 & 0.3 & 1.319 & -0.896 & -0.067 & 0.006 \\
\hline & $\mathrm{C}_{1}$ & & & & & & & & & & & & & & & & \\
\hline \multirow[t]{2}{*}{$\mathrm{X}_{15}$} & $\mathrm{C}_{0}$ & 26 & 355 & & & & & & & & & & & & & -0.098 & -0.126 \\
\hline & $\mathrm{C}_{1}$ & 0.020 & 32 & -0.30 & .015 & 402 & -0.029 & 0.018 & & & $0.06 /$ & & -0.139 & 0.088 & -0.064 & $-\mathbf{0 . 0 4 8}$ & -0.001 \\
\hline \multirow[t]{2}{*}{$X_{16}$} & $\mathrm{C}_{0}$ & $\begin{array}{l}-0.143 \\
0.038\end{array}$ & 184 & 0.411 & 286 & 005 & 0.056 & -0.011 & 0.103 & 0.207 & -0.399 & 0.145 & -0.107 & 0.475 & 0.020 & -0.043 & -0.290 \\
\hline & $\mathrm{C}$ & & -0.017 & -0.007 & 225 & 245 & .038 & -0.005 & & 0.002 & 0.087 & 0.051 & -0.100 & 0.028 & 0.001 & 0.002 & 0.023 \\
\hline
\end{tabular}

Residual effect $\left(\mathrm{C}_{0}\right)$ : 0.114, Residual effect $\left(\mathrm{C}_{1}\right)$ : 0.011, $\mathrm{X}_{1}$ : Plant height, $\mathrm{X}_{2}$ : Panicle length, $\mathrm{X}_{3}$ : No. of panicle/plant, $\mathrm{X}_{4}$ : No. of filled grains/panicle, $\mathrm{X}_{5}$ : 1000-seeds weight, $\mathrm{X}_{6}$ : Husked grain length, $\mathrm{X}_{7}$ : Husked grain width, $\mathrm{X}_{8}$ : Dehusked grain length, $\mathrm{X}_{9}$ : Dehusked grain width, $\mathrm{X}_{10}$ : Shoot length at 45 DAT, $\mathrm{X}_{11}$ : Shoot fresh wt. at 45 DAT, $\mathrm{X}_{12}$ : Shoot dry wt. at 45 DAT, $X_{13}$ : Root length at 45 DAT, $X_{14}$ : Root fresh wt. at 45 DAT, $X_{15}$ : Root dry wt., $X_{16}$ : Amylose content, $C_{0}$ : Control (without Azotobacter inoculation), $\mathrm{C}_{1}$ : Inoculated wit Azotobacter, DAT: Days After Transplanting, Bold figures are direct effects on yield 
condition. The variation in both magnitude and direction in association amongst different characters after seedling inoculation indicate the positive influence of microenvironmental change in root rhizoshpere created by A. chroococcum on the high yielding genotypes, the major constituents of this experiment. Numbers of filled grain per panicle showed significant positive association with 1000 -seed weight and shoot length at 45 DAT in control in addition to the with number of panicles per plant and seed yield per plant. But under inoculated condition, positive and significant association of filled grains per panicle was noted with plant height and amylose content. Positive and significant association of 1000-grains weight was noted with husked grain length and dehusked grain width as well as with shoot fresh weight, root length, root fresh weight and dry weight at 45 DAT in inoculated situation. The erratic pattern of both direction and dimension of association of different plant characters of 45 DAT with other parameters as well as amongst themselves were noted. Shanthakumar et al. (1998) and Gonjales and Ramirez (1998), showed that the significant correlation of 1000seed weight and filled grains per panicle with seed yield which corroborates the present investigation in both situations.

Though the estimates of correlation coefficients mostly indicate the inter-relationship of different characters they do not furnish information on cause and effect. Therefore, path coefficient analysis was done for the purpose.

Table 2 represents the results of path coefficient analyzed based on the genetic correlation coefficient. The highest positive direct effect was contributed by shoot fresh weight followed by root length at 45 DAT in uninoculated condition. But it was highest for 1000grains weight followed by number of panicles per plant under inoculated condition. The change in direction and/or magnitude of direct effects of most of the attributes indicated the influence of inoculum. Plant height, grain yield per plant, 1000-grain weight, grain length, dehusked grain width, shoot length at 45 DAT, shoot dry weight at 45 DAT and root length at 45 DAT also exhibited high positive direct effect on seed yield per plant under control condition. Whereas, panicles per plant, number of filled grains per panicle, dehusked grain length dehusked grain width and shoot length at 45 DAT showed high and positive direct effect on seed yield per plant under inoculated condition. Only number of filled grains per panicle, 1000-seed weight, dehusked grain width and shoot length at 45 DAT showed high positive direct effect under both Azotobacter inoculated and uninoculated conditions. The above results suggested the influence of $A$. chroococcum in alteration of direct effect of different characters on seed yield per plant.

Positive direct effects were noted by Chaubey and Singh (1994) for plant height, number of panicle per plant, number of grains per panicle and 1000-seed weight toward yield. 1000 -seed weight was also considered as the most important character for selection of high yielding genotypes by Shanthakumar et al. (1998), Paul and Sarmah (1997).

The dimensions of residual effect were very low, which indicated the consideration of most of the yield contributing characters. Low residual effect was also reported by Meenakshi et al. (1999) while considering nine yield attributes for path analysis in rainfed rice.

\section{REFERENCES}

Al-Jibouri, H.J., Miller, P.A. and Robinson, H.F. (1958) Genotypic and environmental variances in upland cotton crosses of interspecific origin, Agron Journal 50 633-637

Chakraborty, S., Das, P.K., Guha, B., Barman, B., Sarmah, K.K. (2001) Coheritibility, correlation and path analysis of yield components in Boro rice, Oryza 38(3\&4) 99.101

Chaubey, P.K. and Singh, R.P. (1994) genetic variability, correlation and path analysis of filled componenets in rice, Madras Agricultural Journal 81(9) 438-470

Dean, D. and Jacobson, M.R. (1992) Biochemical genetics of nitrogenase, in: Biological Nitrogen Fixation (eds.) Stacey G, Evans H J, Burris R C and Hall, New York. pp. 763-834

Dewey, D.R. and Lu, K.H. (1959) A correlation and path analysis of components of created wheat grass seed production, Agron Journal 51 515-518

Gonjales, O.M. and Ramirez, R. 1998. Genetic variability and path analysis in rice grown in saline soil. Int. Rice Res. Notes. 23 (3): 19

Ladha, J.K. (1986) Stdies on nitrogen fixation 
by free-living rice plant associated bacteria in wetland rice field, Bionature 6 4758

Meenakshi, T., Ratinam, A.A.D., Backiyarani, S. (1999). Correlation and path analysis of yield and some physiological charactes in rainfed rice. Oryza. 36 (2): $154-156$

Paul, S.K., Sarmah, A.K. (1997). Interrelationship of yield and yield contributing characters in upland ahu rice. J. Agri. Sci. Sco., N.E., India. 10 (2): 164-167

Roger, P. and Ladha, J.K. (1992) Biological nitrogen fixation in wetland rice: estimation contribution to nitrogen balance, Plant Soil 141 41-45

Sadasivam, S. and Manickam, A. (1996) Bio- chemical Methods, $2^{\text {nd }}$ edition, New Age International Pvt. Ltd. Publishers. pp. 1213

Santhakumar, G., Mahadevappa, M. and Rudraradhya (1998). Studies on genetic variability, correlation and path analysis in rice (Oryza sativa L.) over seasons. Karnataka J. Agril. Sci. 11 (1): 67-72

Yanni, Y.G. (1991) Potential of indigenous cyanobacteria to contribute to rice performance under different schedules of nitrogen application, World Journal of $\mathrm{Mi}$ crobiology and Biotechnology 7 48-52

Yogamenakshi, Nadarajan, N. and Ambularmathi, J. (2004) Correlation and path analysis on yield and drought tolerant attributes in rice (Oryza sativa L.) under drought stress, Oryza 41 (3\&4): 68-70 